Widefield Science and Technology for the SKA

SKADS CONFERENCE 2009

S.A. Torchinsky, A. van Ardenne, T. van den Brink-Havinga, A.J.J. van Es, A.J. Faulkner (eds.)

4-6 November 2009, Château de Limelette, Belgium

\title{
SKADS DS2 and Beyond
}

\author{
J.E. Noordam
}

\begin{abstract}
ASTRON, Oude Hoogeveensedijk 4, NL-7991 PD Dwingeloo, The Netherlands
\end{abstract}
\begin{abstract}
The Design Study on Astronomical and Instrumental Simulation (DS2) has been one of the most successful parts of the SKADS program. Although simulation has always been considered important, little effort has been expended in building suitable tools and actually using them. This situation has now changed because of the calibration challenges posed by the next generation of huge radio telescopes. In addition, because of the important role of modelling in 3rd Generation Calibration (3GC), the simulation tools are a natural byproduct of the new processing software. The process is accelerated by a deliberate emphasis on collaborating remotely, for instance by means of the "SKADS Set of Standard Challenges" (SSSC) website operated from Oxford. The latter has also emerged as a prime teaching device for the next generation of radio astronomers. We will discuss the present situation, and stress the importance of a 5-point follow-up program to reap the full harvest of SKADS DS2.
\end{abstract}

\section{Introduction}

The SKADS programme has been very successful in stimulating clever and motivated people in different institutes to work together, and this is due in a large part to the DS2 package (simulation). The world now has an end-to-end simulation infrastructure that can produce simulated data as would be produced by any radio telescope (including SKA, of course), observing any sort of sky (see, in these proceedings, Levrier et al. 2010; Klöckner et al. 2010).

In addition, DS2 has yielded some important by-products. First of all, there is the SKADS/Oxford Set of Standard Challenges (SSSC), which is the combination of a proving ground for new algorithms, and a powerful teaching tool for the new generation of radio astronomers. Secondly, a new style of Marie Curie workshop, designed particularly to have a more lasting impact than the old style (the SSSC has played a role in selecting participants for it). Thirdly, the emergence of a Creative Commons, a web-based community that is addressing the problems of 3rd Generation Calibration (3GC) collectively.

All these by-products have the highly desirable effect of empowering the smaller institutes in the world, by allowing them to participate in the SKA adventure without depriving them of their best talent. This "should bring a smile on the lips of the faceless bureaucrats in Brussels." (NB: When this remark was made at the conference it caused some consternation among our leaders, but rather amused the attending bureaucrat herself.)

\section{DS2 in hindsight}

The DS2 package was divided into two tasks: Sky Simulations (T1) and Intstrumental Simulations (T2). The first was a great success, as reported by Steve Rawlings and others at this conference. On the whole, the subject chimes well with astronomical practice, so people know how to approach it. In addition, the output format is not critical.

In contrast, $\mathrm{T} 2$ was underestimated and undermanned, especially in view of the long and difficult history of the subject. Despite various attempts over the last decades, nobody has had the will, the resources or the ideas to generate an instrumenta simulation package that could be used as a starting point for the kind of simulations that are needed for a telescope like SKA.

Fortunately, the SKADS culture of bringing people in contact with each other saved the day. First of all, some new ideas greatly simplified the discussion about array configurations, as reported by others at this conference (Bolton et al. 2010; Lobanov et al. 2010; Ford et al. 2010). And secondly, the MeqTrees package, which was developed originally for LOFAR, turned out to offer the desired instrumental simulation as a by-product of its normal operation. This meant that little extra investment was needed to generate the promised simulation pipeline.

However, it has become clear that the follow-up program for DS2 should be partitioned somewhat differently. Therefore, package leader Thijs van de Hulst has proposed the following at recent SKADS meetings:

- P1: Sky Simulations

- P2: Put these into processable form (Global Sky Model)

- P3: Generate corrupted uv-data

- P4: Calibration (i.e. retrieve the simulated instr. errors)

- P5: Comparison with the input

The five parts represent roughly the same amount of work, which can be assigned to different kinds of people. For instance, only P1 and P5 require astronomers. The abovementioned SSSC, Creative Commons, new-style Marie-Curie workshops and empowerment of smaller institutes can (and should!) all play a role in this process.

\section{Some closing remarks}

It is clear that SKADS DS2 is a Big Success. Not only it has, rather unexpectedly, achieved all it set out to achieve, but it has produced much more. Apart from the 4 main by-products mentioned above, there are some others that should have a considerable impact on the way people work together:

1. The so-called "Purr Log" is a, deceptively simple, but remarkably effective way to generate a detailed report about 
a data reduction project. It is easier to generate a Purr Log than to keep notes on scraps of paper, and the result is directly available (as html) for dissemination among collaborators and critics.

2. It is uncontroversial by now that the best way to model a radio telescope is by means of the full-polarization Measurement Equation (M.E.), which has a sucession of $2 \times 2$ Jones matrices for instrumental effects. The MeqTrees package has been designed to implement an arbitrary M.E., so it can model any telescope.

3. The "Tree Time Unit" (TTU) is the elapsed time between having a new idea, implementing it in MeqTrees, and reducing the data to the point of having an image to look at. Rather than the usual time of weeks, months, years, or even decades to get new ideas implemented, the TTU is less than a day.

4. New ideas are implemented in the form of Tree Definition Language (TDL) scripts. Since these are just Python scripts (ascii), they can be easily exchanged between collaborators, together with a Purr Log.

Thus, in addition to the DS2 simulation pipeline, we now have a Common Language (the M.E.) and Common Tools (Purr logs and TDL scripts), and meeting places like the Creative Commons, the SSSC and the new-style Marie Curie workshops, and the TTU. We have also established that there are a lot of clever people at the smaller institutes, who are hungry to join the SKA adventure. The combination of these factors has the potential to increase the rate of evolution of radio astronomical data reduction software by several orders of magnitude, while generating a new generation of users. Both are urgently needed, because the new telescopes are being built right now, and we are far from ready to exploit their huge (and hugely expensive) potential.

Summarizing, it is not often that so many things fall into place in a single program. But it DOES need the oxygen of a little funding. The conclusion is that it would be a tragedy not to follow up on DS2.

Acknowledgements. I am very grateful to the SKADS Management Team, especially Steve Torchinsky who wrote these acknowledgements. Many people have contributed over the last decade or so to the development of 3rd Generation Calibration, most of whom have had enormous patience with my philosophical meanderings and my good natured banter. I particularly want to thank Jaap Bregman, and of course, Oleg Smirnov, the genius behind the implementation of MeqTree, as well as the new generation of radio astronomers represented first of all by Ian Heywood. The SKADS Marie-Curie Conferences and Training program was funded by the European Commission Framework Progam 6 Marie-Curie Actions Contract No. 46095, and I wholeheartedly acknowledge the great foresight of our EC progam manager, Elena Righi, who fully supported this program.

\section{References}

Bolton, R.C., et al., "Figures of merit for SKA scale configurations," 2010, in Proc. Wide Field Science and Technology for the SKA, Limelette, Belgium, S.A. Torchinsky et al. (eds), ASTRON, ISBN 978-90-805434-5-4

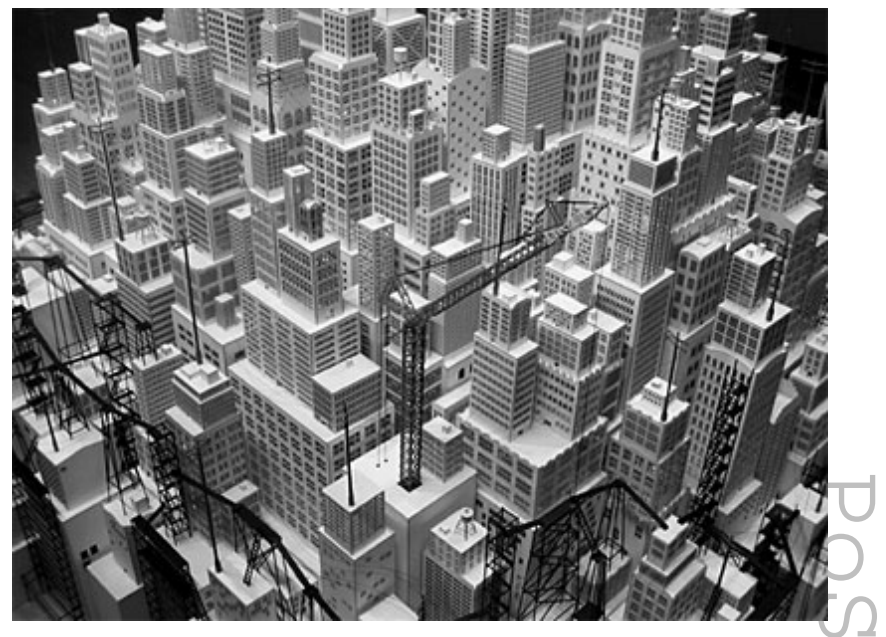

Fig. 1: Even though this image might remind some people of Metropolis by Fritz Lang, it is an apt metaphore of what we are trying to do. Progress in any field is greatest when many people get involved, influencing but not constraining each other, building towards a loosely defined common goal. The SKADS DS2 package has greatly improved the conditions for this, with a common language (the Measurement Equation), common tools (MeqTrees, Purr logs), and various web-based meeting places (SSSC, Creative Commons). As a byproduct, clever but isolated and hungry people in the smaller institutes all over Europe (or indeed the World) will now be able to join the great SKA adventure without being drained away.

Ford, D., et al., "The SKA Cost/Performance Tool: A Hierarchical SKA Modelling Tool," 2010, in Proc. Wide Field Science and Technology for the SKA, Limelette, Belgium, S.A. Torchinsky et al. (eds), ASTRON, ISBN 978 90-805434-5-4

Levrier, F., et al., "Mapping the SKA Simulated Skies with the $S^{3}$-Tools," 2010, in Proc. Wide Field Science and Technology for the SKA, Limelette, Belgium, S.A. Torchinsky et al. (eds), ASTRON, ISBN 978-90805434-5-4

Klöckner, H.R., et al., "SKA HI end2end simulation," 2010, in Proc. Wide Field Science and Technology for the SKA, Limelette, Belgium, S.A. Torchinsky et al. (eds), ASTRON, ISBN 978-90-805434-5-4

Lobanov, A., et al., "SKADS Array Configuration Study," 2010, in Proc. Wide Field Science and Technology for the SKA, Limelette, Belgium, S.A. Torchinsky et al. (eds), ASTRON, ISBN 978-90-805434-5-4 